Ann. Biol. anim. Bioch. Biophys., 1977, 17 (4), 499-502.

\title{
Commande bulbaire de la motricité linguale chez le mouton
}

\author{
par M. FALEMPIN *, Y. VAN BESIEN, J. P. ROUSSEAU \\ Laboraioire de Neurophysiologie végétative, SN4, \\ Université des Sciences et Techniques de Lille, \\ 59650 Villeneuve d'Ascq \\ * Faculté de Chirurgie dentaire, Lille.
}

Summary. Activity of hypoglossal motor fibres innervating the sheep tongue.

The left hypoglossal and spinal accessory nerves (XIIth and Xlth cranial nerves) were sectioned in sheep. The central stump of the hypoglossal nerve was sutured end to end to the caudal stump of the spinal accessory nerve. Six to nine months after the nervous crossed-suture procedure, the activity of the reinnervated fibres of the sterno-cleidomastoidus and trapezius muscles indicated hypoglossal motor fibre activity. Using electromyography in the unanaesthetized sheep, the discharge of hypoglossal motoneurones originally innervating the tongue muscles was studied.

Type I units were recorded during bolus chewing ; they fired in bursts of 2-8 action potentials ( $30-150 \mathrm{~ms}$ duration) between the successive electromyograms of the masseter when the mouth opened. Potential frequency was $70 \mathrm{AP} / \mathrm{s}$. These unit activities were also elicited by factile stimulation of the lips or by stretching the tongue. Type II units were silent during chewing; they fired in a long burst of action potentials (200-1 $500 \mathrm{~ms})$ at the beginning of swallowing when the mylohyoidus contracted. These results are discussed.

Le nerf grand hypoglosse est moteur ef innerve la musculature complexe de la langue. On sait que l'activité des neurones du noyau bulbaire de l'hypoglosse est rapidement modifiée par la stimulation afférente des nerfs lingual et glossopharyngien (Blom, 1960 ; Porter, 1965). Les modalités de leur décharge pendant la prise alimentaire et la déglutition sont par contre mal connues. Pour aborder cette étude chez l'animal éveillé, nous utilisons la technique des sutures nerveuses hétérogènes croisées. Rappelons que par cet artifice expérimental, Dussardier (1963), Roman (1966), Miolan et Roman (1974) font réinnerver le diaphragme ou les muscles trapèze ef mastoïdohuméral par des axones moteurs vagaux. Les activités électromyographiques de ces muscles reflètent l'activité de neurones vagaux qui innervaient d̀ l'origine l'œsophage et l'estomac.

Nous suturons chez 2 moutons l'extrémité centrale du nerf hypoglosse gauche 
sectionné à l'extrémité périphérique du nerf spinal, moteur du muscle mastoïdohuméral. Six à neuf mois après l'intervention, on recueille l'activité des fibres musculaires réinnervées à l'aide d'aiguilles de Bronk : l'activité d'une unité motrice traduit l'activation d'un motoneurone de l'hypoglosse. Pour situer cette activité par rapport à des événements moteurs digestifs, nous enregistrons en même temps l'électromyogramme des muscles masséter et mylohyoïdien, qui sont innervés par le nerf trijumeau. Le masséter, élévateur de la mandibule, est actif pendant la fermeture de la cavité buccale. Le muscle mylohyoïdien participe en tant qu'abaisseur de la mâchoire à son ouverture. Il mobilise en plus l'ensemble os hyoïdien - langue au début de la déglutition et projette le massif lingual contre la voûte du palais.

\section{Résultats.}

Nous enregistrons l'activité de nombreuses unités motrices pendant la mastication du bol alimentaire. Chaque unité pulse en une succession d'une cinquantaine de salves rythmées sur la cadence des coups de mâchoires. La figure 1 montre que les salves apparaissent dans les intervalles de temps qui séparent deux activités

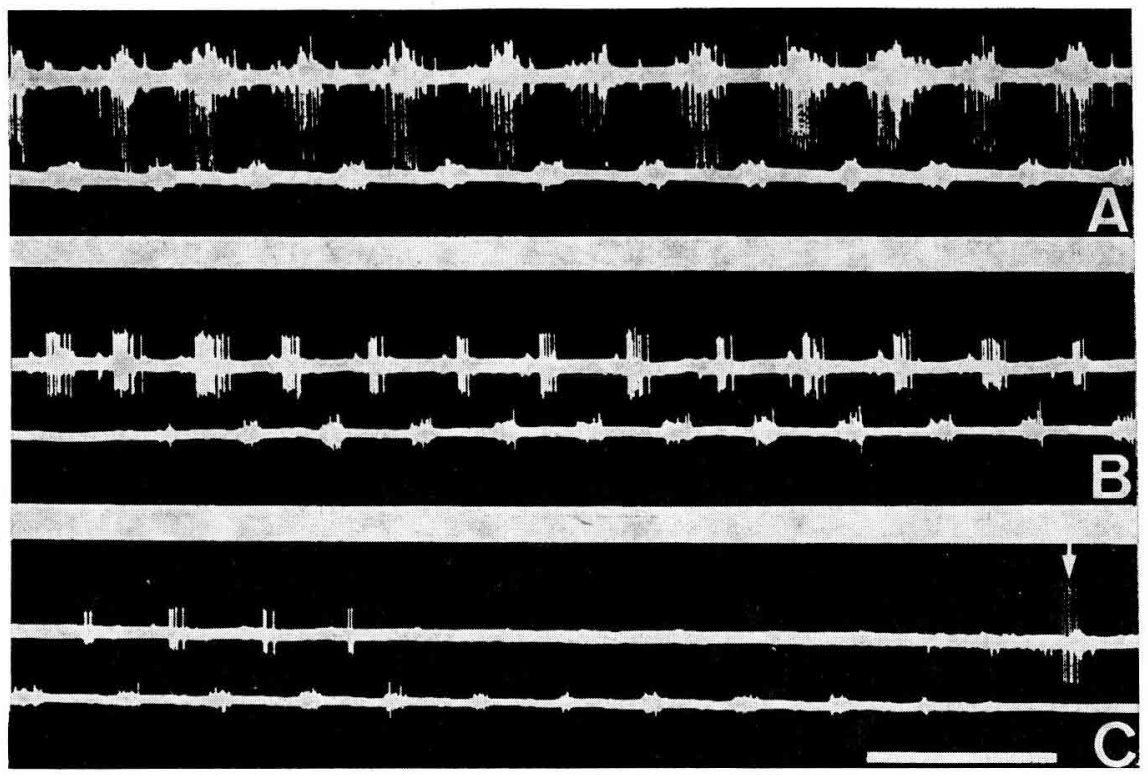

FIG. 1. Enregistrement de l'activité de fibres musculaires réinnervées par les motoneurones de l'hypoglosse, pendant la mastication. La $1^{\text {re }}$ ligne des tracés $A, B$ et $C$ correspond à l'enregistrement de l'EMG du muscle mastoïdo-huméral réinnervé, la seconde ligne à celui de l'EMG du masseter. En $A$, on recueille plusieurs unités qui pulsent en salves, entre chaque activité du masseter.

En $B$ et $C$, on enregistre l'activité d'une seule et même unité, pendant la mastication. Noter la variation du nombre de potentiels au sein de chaque salve et le silence de l'unité lors des 7 derniers coups de mâchoires.

En $C$, on recueille une unité (flèche) contemporaine de la fin de la mastication au moment de la déglutition du bol.

Etalonnage : $1 \mathrm{~s}$. 
successives du muscle masséter. Elles sont contemporaines de l'activité du muscle mylohyoïdien. Elles durent de 30 à $150 \mathrm{~ms}$ et sont constituées de 2 à 8 potentiels d'action dont la fréquence moyenne de décharge est de 70 par s. La décharge de ces unités évolue au cours du temps, tout au long de la mastication du bol. Si la fréquence des potentiels demeure comparable d'une salve à la suivante, leur nombre au sein des salves successives varie de façon périodique. La majorité des unités cesse de pulser lors des derniers coups de mâchoires qui précèdent la déglutition (fig. $1 \mathrm{C}$ ).

La stimulation tactile des lèvres provoque la décharge de ces unités en bouffées irrégulières de potentiels dont la fréquence reste élevée (fig. 2 A-B). Lorsqu'on maintient la langue tirée vers l'avant, on observe en premier lieu l'apparition d'une décharge de type tonique pendant une à deux secondes, puis secondairement des salves d'activité phasique (fig. 2 C-D) identiques à celles enregistrées au cours de la stimulation labiale. La décharge tonique est contemporaine de la résistance musculaire ressentie dès que l'on exerce la traction de la langue ; cette résistance cesse avec l'arrêł de l'activité tonique; puis des mouvements rapides de rétraction correspondent à l'apparition des salves phasiques.

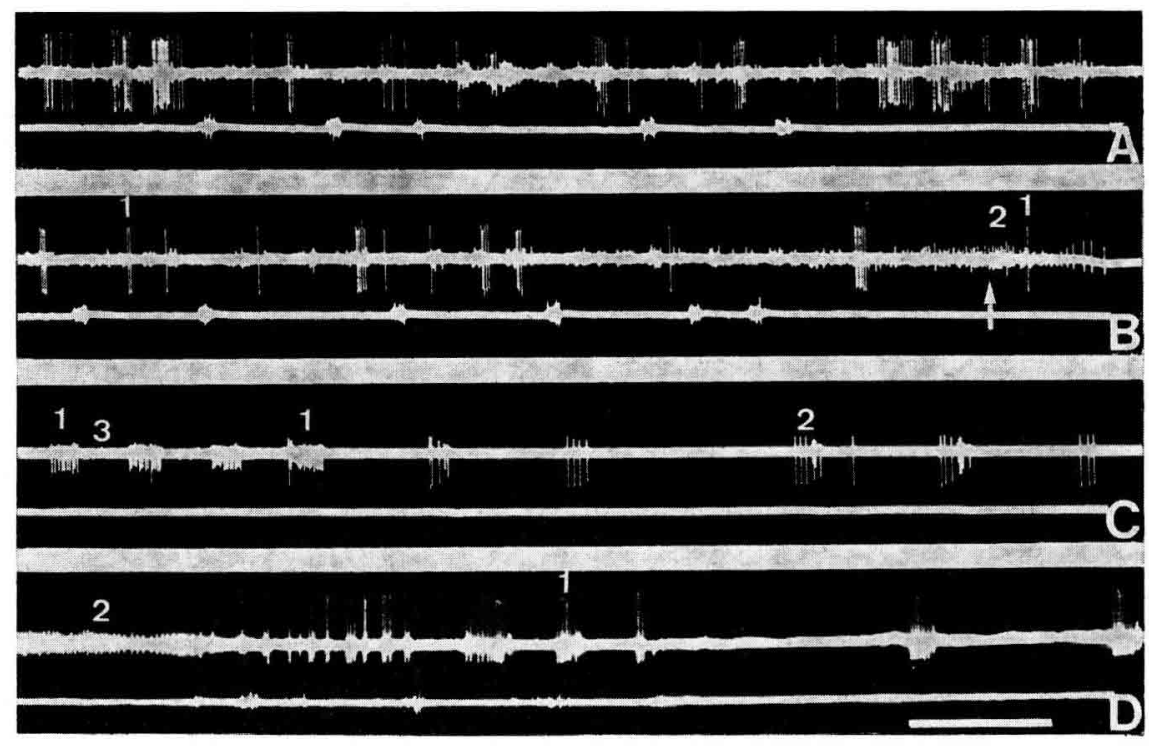

FIG. 2. - Enregistrement de l'activité de fibres musculaires réinnervées par les motoneurones de l'hypoglosse, au cours de divers types de stimulations périphériques.

La 1 re ligne des tracés A, B, C ef D correspond à l'enregistrement de l'EMG du muscle mastoïdohuméral réinnervé, la seconde ligne à celui de l'EMG du masseter.

En $A$ ef $B$, on recueille une unité (1) de grande amplitude qui pulse irrégulièrement pendant la durée d'une stimulation tactile portée sur les lèvres de l'animal. L'unité (2) de plus faible amplitude est contemporaine d'une déglutition à vide (flèche). En $C$ et $D$, on recueille plusieurs unités pendant la durée d'une traction exercée sur la langue. La décharge d'unités toniques est enregistrée dès le début de l'étirement : l'unité (3) en C dépasse à peine le bruit de fond, mais l'unité (2) est bien visible en $D$. Des activités en salves (unités 1 et 2 en $C$, unité 1 en D) apparaissent plus ou moins tardivement alors que le stimulus est maintenu.

Etalonnage : $1 \mathrm{~s}$. 
Enfin, certaines unités, silencieuses pendant la mastication, sont recueillies seulement au début de la déglutition (fig. $1 \mathrm{C}$ et fig. $2 \mathrm{~B}$ ). Elles pulsent en une salve de 0,2 à 1,5 secondes de durée, contemporaine de l'activité électromyographique de déglutition du muscle mylohyoïdien.

\section{Discussion.}

Deux types d'unités sont recueillis pendant la prise alimentaire. Les unités les plus couramment rencontrées sont actives pendant la mastication. Comme elles apparaissent en salves entre les contractions successives du masséter, elles traduisent vraisemblablement l'activation de neurones de l'hypoglosse qui, à l'origine, commandaient les mouvements de la langue lors des périodes d'ouverture de la bouche. Les unités actives après l'arrêt de la mastication reflètent plutôt l'activité de neurones qui normalement sont responsables de la projection du massif lingual contre la voûte du palais, à la fin du temps buccal de la déglutition. On ne peut pas toutefois identifier les muscles de la langue qu'innervaient ces neurones; il est malaisé d'enregistrer sélectivement sur l'animal éveillé l'activité, qui pourrait servir de référence, d'un muscle donné intrinsèque ou extrinsèque de la langue. Les muscles du côté gauche sont par ailleurs partiellement dégénérés du faił de leur dénervation, bien que l'animal ait récupéré une motricité linguale apparemment satisfaisante. La langue est en effel constituée de structures musculaires paires, à la différence de l'œsophage et de l'estomac, organes impairs innervés par un nerf droit et un nerf gauche dont on peut supposer qu'ils transmettent de façon synchrone le même ordre moteur (Dussardier, 1960). Or, lors de déplacements latéraux de la langue, il est vraisemblable que l'activité du nerf hypoglosse gauche n'est pas rigoureusement identique à celle de l'hypoglosse droit. Il faudra tenir compte de cette restriction qui peut limiter les avantages que l'on peut attendre de l'emploi de la technique des sutures nerveuses dans l'étude de la commande bulbaire de la motricité linguale chez l'animal éveillé.

Commission CNERNA Digestion-Absorption, Tours, 13 novembre 1976.

\section{Références}

BLOM S., 1960. Afferent influences on tongue muscle activity. A morphological and physiological study in the cat. Acta physiol. scand., 49, Suppl. 170, 97 p.

DUSSARDIER M., 1960. Recherches sur le contrôle bulbaire de la motricité gastrique chez les Ruminants. Thèse Doct. ès-Sci. Nat., Paris, 199 p.

DUSSARDIER M., 1963. Réinnervation d'un muscle strié par les fibres préganglionnaires parasympathiques. Application à l'enregistrement de l'activité des fibres efférentes vagales chez l'animal éveillé. Ann. Biol. anim., Bioch., Biophys., 3, 405-425.

MIOLAN J. P., ROMAN C., 1974. Décharge unitaire des fibres vagales efférentes lors de la relaxation réceptive de l'estomac du chien. J. Physiol., Paris, 68, 693-704.

PORTER R., 1965. Synaptic potentials in hypoglossal motoneurons. J. Physiol., Lond., 180, 209-226.

ROMAN C., 1966. Contrôle nerveux du péristaltisme œsophagien. J. Physiol, Paris, 58, 79-108. 